\title{
Relaxations of mixed integer sets from lattice-free polyhedra
}

\section{Journal Article}

Author(s):

Del Pia, Alberto; Weismantel, Robert

Publication date:

2012-09

Permanent link:

https://doi.org/10.3929/ethz-b-000051191

Rights / license:

In Copyright - Non-Commercial Use Permitted

Originally published in:

4OR 10(3), https://doi.org/10.1007/s10288-012-0198-8 


\title{
Relaxations of mixed integer sets from lattice-free polyhedra
}

\author{
Alberto Del Pia • Robert Weismantel
}

Received: 29 September 2011 / Revised: 17 January 2012 / Published online: 15 February 2012

(C) Springer-Verlag 2012

\begin{abstract}
This paper gives an introduction to a recently established link between the geometry of numbers and mixed integer optimization. The main focus is to provide a review of families of lattice-free polyhedra and their use in a disjunctive programming approach. The use of lattice-free polyhedra in the context of deriving and explaining cutting planes for mixed integer programs is not only mathematically interesting, but it leads to some fundamental new discoveries, such as an understanding under which conditions cutting planes algorithms converge finitely.
\end{abstract}

Keywords Mixed integer programming - Cutting planes - Disjunctive programming · Lattice-free polyhedra

MSC classification (2000) Primary 90C11; Secondary 90C10

\section{Introduction}

Cutting plane techniques have always been one of the prominent topics in the theory of integer and mixed integer programming. We focus here on a treatment of some of the novel developments in this direction. Herewith we pursue the goal of understanding geometric principles that allow cutting plane algorithms to terminate finitely. To be more specific, the set of feasible solutions of a mixed integer programming problem attains the form

$$
P \cap\left(\mathbb{Z}^{p} \times \mathbb{R}^{q}\right)
$$

\footnotetext{
A. Del Pia $(\varangle) \cdot$ R. Weismantel

Department of Mathematics, IFOR, ETH Zürich, Rämistrasse 101, 8092 Zurich, Switzerland e-mail: alberto.del.pia@ifor.math.ethz.ch

R. Weismantel

e-mail: robert.weismantel@ifor.math.ethz.ch
} 

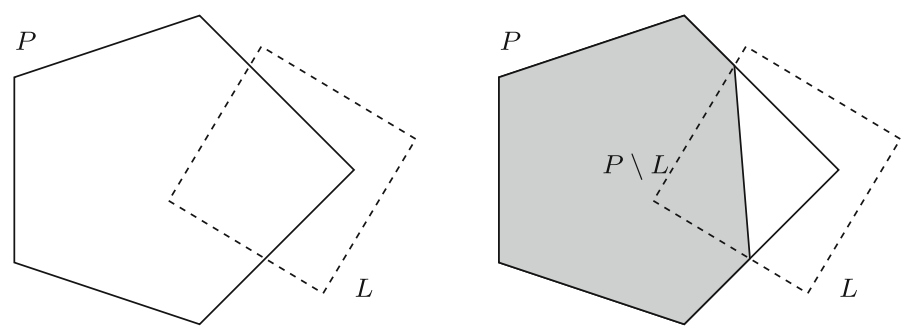

Fig. 1 On the left polyhedra $P$ and $L$. On the right, the resulting polyhedron $P \backslash L$

where $P$ is a polyhedron in $\mathbb{R}^{p+q}$, and $p, q \in \mathbb{N}$, with $p \geq 1, q \geq 0$. By a polyhedron we always mean the solution set of a finite system of linear inequalities. We call a bounded polyhedron a polytope.

The mixed integer hull of $P$, that we denote by $P_{I}$, is the set

$$
\operatorname{conv}\left(P \cap\left(\mathbb{Z}^{p} \times \mathbb{R}^{q}\right)\right),
$$

where we denote by "conv" the convex hull. If $q=0$ we refer to the mixed integer hull as the integer hull. In this survey we always denote by $z$ the variables in $\mathbb{R}^{n}=\mathbb{R}^{p+q}$, by $x$ the variables in $\mathbb{R}^{p}$, which are the variables subject to integrality constraints, and by $y$ the variables in $\mathbb{R}^{q}$, which are the variables not required to be integer. We recall that a polyhedron is called rational if it is the solution set of a finite system of linear inequalities with rational data. The following follows from Meyer (see Meyer 1974; Schrijver 1986, Section 16.7):

Theorem 1 Let $P$ be a rational polyhedron in $\mathbb{R}^{p+q}$. Then $P_{I}$ is a rational polyhedron.

Most of the research in mixed integer programming is dedicated to the question how to derive inequalities from a description of $P$ that are satisfied by all the points in $P \cap\left(\mathbb{Z}^{p} \times \mathbb{R}^{q}\right)$. Such inequalities naturally define relaxations of $P_{I}$ in form of polyhedra that are contained in $P$ and that contain $P_{I}$.

In order to obtain polyhedra contained in $P$ and that contain $P_{I}$ we use an operator introduced in Andersen et al. (2010) that may be viewed as a special disjunctive programming approach invented by Balas (1998).

More formally, given polyhedra $P, L \subseteq \mathbb{R}^{p+q}$, we denote by $P \backslash L$ the smallest closed convex set that contains $P \backslash$ int $L$, where "int" denotes the interior (see Fig. 1). Hence if we denote by "conv" the closed convex hull,

$$
P \backslash L:=\overline{\operatorname{conv}}(P \backslash \operatorname{int} L) .
$$

It is easy to see that given polyhedra $P, L \subseteq \mathbb{R}^{p+q}$, then $P \backslash L \subseteq P$. It will turn out that the set $P \backslash L$ is again polyhedral. This implies that such an operation can be also used iteratively. The question arises which polyhedra $L$ one should use in order to approximate $P_{I}$ sufficiently well. This is the central question of our survey paper.

It is convenient to extend the definition of $P \backslash L$ for polyhedra $P \subseteq \mathbb{R}^{p+q}, L \subseteq \mathbb{R}^{p}$. In this case,

$$
P \backslash L:=P \backslash\left(L \times \mathbb{R}^{q}\right) .
$$




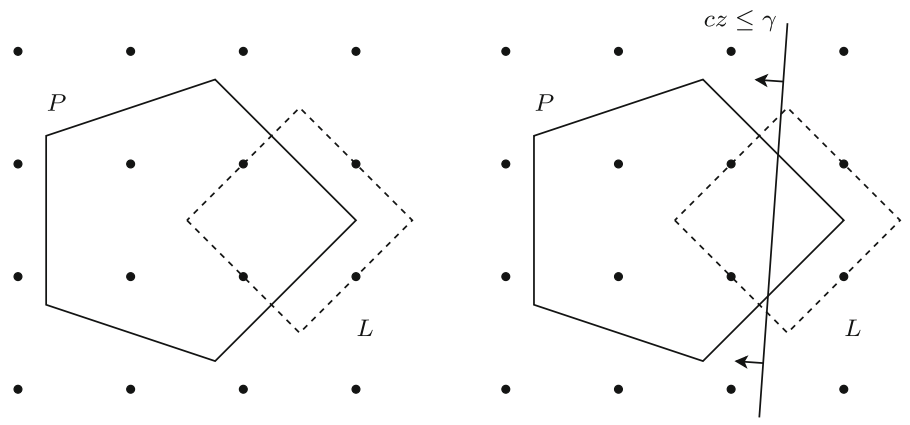

Fig. 2 On the left polyhedra $P$ and $L$. On the right, an $L$-disjunctive cut for $P$
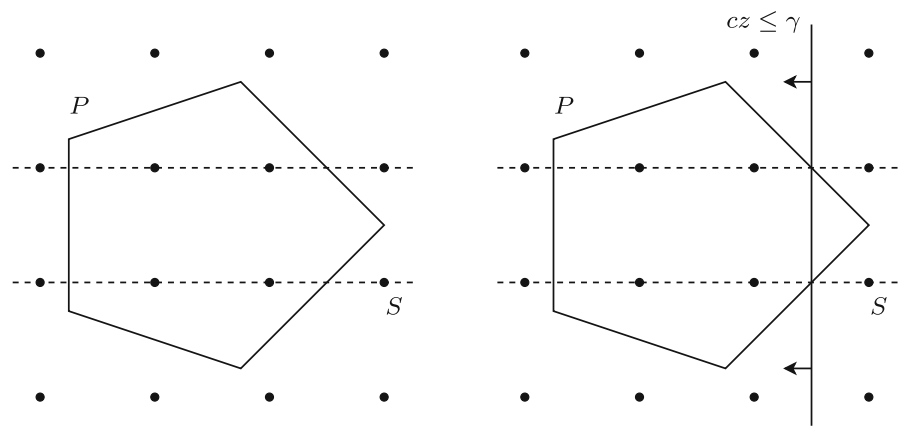

Fig. 3 On the left polyhedra $P$ and $S$. On the right, a split cut for $P$

We will show that in order to generate valid inequalities for $P_{I}$, we will have to use lattice-free polyhedra $L \subseteq \mathbb{R}^{p}$, i.e. polyhedra that do not contain points in $\mathbb{Z}^{p}$ in their interior. In this case, the new set $P \backslash L$ is indeed a relaxation of $P_{I}$ and, in general, a better approximation of the mixed integer hull $P_{I}$ compared to $P$. The new inequalities that arise from this operation are disjunctive cuts that were first introduced by Balas in the 1970's (Balas 1998, 1979). Given a polyhedron $P \subseteq \mathbb{R}^{p+q}$, and a lattice-free polyhedron $L=\left\{x \in \mathbb{R}^{p}: a^{i} x \leq \beta^{i}, i=1, \ldots, k\right\}$, an inequality $c z \leq \gamma$ is called an $(L-)$ disjunctive cut for $P$ if $c z \leq \gamma$ is valid for every set

$$
\left\{z \in P: a^{i} x \geq \beta^{i}\right\}, \quad i=1, \ldots, k
$$

(See Fig. 2.) It follows easily that $c z \leq \gamma$ is an $L$-disjunctive cut for $P$ if and only if $c z \leq \gamma$ is valid for $P \backslash L$.

The simplest family of lattice-free polyhedra are the strips. A polyhedron $S \subseteq \mathbb{R}^{p}$ is called a strip if $S=\left\{x \in \mathbb{R}^{p}: \beta \leq a x \leq \beta+1\right\}$ where $a \in \mathbb{Z}^{p}, \beta \in \mathbb{Z}$, and the greatest common divisor of the entries in $a$ is 1 . The disjunctive cuts corresponding to strips are called split cuts, and were introduced by Cook et al. (1990) (see Fig. 3). Split cuts have been extensively studied in the literature. Split cuts are equivalent (Nemhauser and Wolsey 1990) to Gomory's mixed integer (GMI) cuts (Gomory 1963) and to mixed integer rounding cuts (MIR) (Marchand and Wolsey 2001). Most 
of the cutting planes used in practice for mixed integer programming problems are special classes of split cuts, like for example lift-and-project cuts (Balas et al. 1993).

A recent paper by Andersen et al. (2007) inspired the community to study disjunctive cuts for a special relaxation of mixed integer linear sets in tableau form. This relaxation is called the relaxed corner polyhedron, in which the integrality requirement of all nonbasic variables is neglected. (This is in contrast to the corner polyhedron that was introduced by Gomory 1965, 1969. In fact in the latter model a subset of the nonbasic variables can be required to attain integer values.)

In the relaxed corner polyhedron the integer variables are expressed in terms of the remaining variables as follows:

$$
\begin{aligned}
& x=f+\sum_{j=1}^{q} r^{j} y_{j} \\
& x \in \mathbb{Z}^{p} \\
& y \in \mathbb{R}_{+}^{q} .
\end{aligned}
$$

This model is a natural relaxation of a general mixed integer linear set and therefore it can be used to generate cutting planes for the original mixed integer set. In this special setting disjunctive cuts take the form of intersection cuts, a simpler type of cutting planes introduced by Balas (1971). We refer to Conforti et al. (2011a) for a survey on the relaxed corner polyhedron and intersection cuts.

This is just a very brief summary of some of the newer developments in cutting plane theory. We are conscious of the fact that this treatment is by no means complete and it does not represent all important developments.

The main focus of this survey, though, is to provide a basic mathematical understanding of convergence and the issue of finiteness of cutting plane algorithms. This issue has only very marginal ties to recent research on computational aspects of the topic. This is one excuse of ours that we do not deepen the subject above further.

The survey is organized as follows. In Sect. 2 we study the set $P \backslash L$ and some of its properties. In Sect. 3 we introduce lattice-free polyhedra, and we see how $P \backslash L$ can be used in the context of mixed integer programming. In Sect. 4 we focus on the $\mathcal{L}$-closure $\mathcal{L}(P)$ of $P_{I}$, that is the intersection of many sets $P \backslash L$, where $L$ varies in a family $\mathcal{L}$ of lattice-free polyhedra. In Sect. 5 we study convergence results when we are allowed to use the $\mathcal{L}$-closure recursively, i.e. if we are allowed to take closures of closures. Finally, in Sect. 6 we introduce the concept of rank with the perspective of defining cutting plane proofs.

The careful reader might have noticed that so far we have been considering mainly the case where the given polyhedron $P$ is described by means of rational data. This assumption has been made since in the general (non rational) case, the mixed integer hull of a polyhedron is not necessarily a polyhedron. This can be seen by analyzing the integer hull of $P:=\left\{x \in \mathbb{R}^{2}: x_{1} \geq 0, x_{1}-\sqrt{2} x_{2} \geq 0\right\}$. Note though that if $P$ is a polytope (even in irrational data), then it is easy to see that $P_{I}$ is a rational polytope. The non rational case, even if interesting from a theoretical point of view, is in practice much less appealing. Even if the given data are not rationals, a suitable rational approximation can always be used instead. This is the reason why in the remainder of this survey we focus exclusively on a study of the rational case. 
The following notation will be used throughout this survey. Given a polyhedron $P=\left\{(z, w) \in \mathbb{R}^{n+d}: A z+G w \leq b\right\}$, we denote with $\operatorname{proj}_{z} P \subseteq \mathbb{R}^{n}$ the orthogonal projection of $P$ onto the space of the $z$-variables. More precisely $\operatorname{proj}_{z} P:=\left\{z \in \mathbb{R}^{n}\right.$ : $\left.\exists w \in \mathbb{R}^{d}, A z+G w \leq b\right\}$. It is a well-known fact that $\operatorname{proj}_{z} P$ is a polyhedron. We denote by "cone" the conic hull, by "relint" the relative interior, and by "lin.space" the lineality space. We always assume that rational numbers, vectors, and matrices are given in their usual binary encoding (see for example Schrijver 1986, Chapter 2). If not otherwise specified, polyhedra are given by means of an outer description.

\section{Union of polyhedra}

The main objective of this section is to study the closed convex hull of a finite union of polyhedra.

Given polyhedra $P^{i}, i=1, \ldots, k$, in this section we consider the smallest closed convex set that contains all polyhedra $P^{i}, i=1, \ldots, k$. This set is defined as

$$
\overline{\operatorname{conv}} \bigcup_{i=1}^{k} P^{i}
$$

The following observation follows easily from the Hahn-Banach separation Theorem (see for example Rockafellar 1970).

Observation 2 Given polyhedra $P^{i}, i=1, \ldots, k$, the set $(1)$ is the intersection of all the half-spaces containing $\bigcup_{i=1}^{k} P^{i}$.

Note that the topological closure is required in (1) in order to get a closed set, as the convex hull of a union of polyhedra is not always closed. As an example consider polyhedra $P^{1}:=\left\{z \in \mathbb{R}^{2}: z_{2}=0\right\}$, and $P^{2}:=\left\{(0,1) \in \mathbb{R}^{2}\right\}$.

By Minkowski-Weil's Theorem (see for example Schrijver 1986), for every polyhedron $P^{i}$ there exist a polytope $Q^{i}$ and a polyhedral cone $C^{i}$ such that $P^{i}=Q^{i}+C^{i}$. The following theorem can be found in Conforti et al. (2010b, Theorem 11.17) and characterizes the set (1).

Theorem 3 Let $P^{i}=Q^{i}+C^{i}, i=1, \ldots, k$ be nonempty polyhedra. Then $\overline{\mathrm{conv}} \bigcup_{i=1}^{k} P^{i}$ is the polyhedron

$$
\overline{\operatorname{conv}} \bigcup_{i=1}^{k} P^{i}=\operatorname{conv} \bigcup_{i=1}^{k} Q^{i}+\operatorname{cone} \bigcup_{i=1}^{k} C^{i}
$$

In particular Theorem 3 states that the set (1) is polyhedral.

Given polyhedra $P^{i} \subseteq \mathbb{R}^{n}, i=1, \ldots, k$, the polyhedron $\overline{\operatorname{conv}} \bigcup_{i=1}^{k} P^{i}$ can be very complicated, as the number of its facets can grow exponentially with $n$ already for $k=2$ (Benoy et al. 2005). This means that in practice we cannot generally get a complete description of $\overline{\text { conv }} \bigcup_{i=1}^{k} P^{i}$. However we will see in the next section that it is possible to efficiently describe $\overline{\operatorname{conv}} \bigcup_{i=1}^{k} P^{i}$ in a higher dimensional space. 


\subsection{An extended formulation}

Given polyhedra $P^{i} \subseteq \mathbb{R}^{n}, i=1, \ldots, k$, Balas $(1998,1985)$ showed that $\overline{\text { conv }} \bigcup_{i=1}^{k} P^{i}$ is the projection of a polyhedron in a higher dimensional space, with polynomially many variables and constraints.

For $i=1, \ldots, k$, let $P^{i}=\left\{z \in \mathbb{R}^{n}: A^{i} z \leq b^{i}\right\}$ be nonempty polyhedra. Consider the following system of linear inequalities:

$$
\begin{aligned}
& A^{i} z^{i}-b^{i} \lambda^{i} \leq 0 \quad i=1, \ldots, k \\
& z-\sum_{i=1}^{k} z^{i}=0 \\
& \sum_{i=1}^{k} \lambda^{i}=1 \\
& \lambda^{i} \geq 0 \quad i=1, \ldots, k .
\end{aligned}
$$

Note that the system $(2-5)$ has $n+(n+1) k$ variables, which are $z \in \mathbb{R}^{n}$, and $z^{i} \in \mathbb{R}^{n}, \lambda^{i} \in \mathbb{R}$, for $i=1, \ldots, k$. Moreover the system (2-5) has $n+1$ equations, and other $\sum_{i=1}^{k} m^{i}+k$ inequalities, where $m^{i}$ is the number of rows of $A^{i}$. The following is Balas' theorem on union of polyhedra.

Theorem 4 Let $P^{i}=\left\{z \in \mathbb{R}^{n}: A^{i} z \leq b^{i}\right\}, i=1, \ldots, k$, be nonempty polyhedra, and let $\tilde{P} \subseteq \mathbb{R}^{n+(n+1) k}$ be the polyhedron defined by the system (2-5). Then

$$
\overline{\operatorname{conv}} \bigcup_{i=1}^{k} P^{i}=\underset{z}{\operatorname{proj}} \tilde{P} .
$$

Theorem 4 does not hold, in the presented form, if some polyhedra among $P^{i}, i=$ $1, \ldots, k$, are empty. However sometimes it is useful to consider directly this more general case. We refer to Conforti et al. (2010b, Theorem 11.18) for a similar but more general statement.

Theorem 4 is a basic ingredient for efficiently optimizing over (1) by solving a single linear programming problem. The trick is namely to optimize the given objective function not in the original space of the $z$ variables, but in the extended space where $\tilde{P}$ lies. Since linear programming problems can be solved in polynomial time (see Khachiyan 1979, 1980), the following holds:

Corollary 5 Given polyhedra $P^{i}, i=1, \ldots, k$, it is possible to optimize over $\overline{\mathrm{conv}} \bigcup_{i=1}^{k} P^{i}$ in polynomial time.

\subsection{A convexification operation}

In this survey, a fundamental role is played by the following object. Given polyhedra $P, L \subseteq \mathbb{R}^{n}$, we denote by $P \backslash L$ the smallest closed convex set that contains $P \backslash \operatorname{int} L$, i.e. 


$$
P \backslash L:=\overline{\operatorname{conv}}(P \backslash \operatorname{int} L) .
$$

The polyhedrality of $L$ implies that there exist inequalities $a^{i} z \leq \beta^{i}, i=1, \ldots, k$, such that $L=\left\{z \in \mathbb{R}^{n}: a^{i} z \leq \beta^{i}, i=1, \ldots, k\right\}$. It follows that

$$
P \backslash \operatorname{int} L=\bigcup_{i=1}^{k}\left(P \cap\left\{z \in \mathbb{R}^{n}: a^{i} z \geq \beta^{i}\right\}\right) \text {. }
$$

In particular:

Observation 6 Let $P$ and $L$ be polyhedra. Then $P \backslash \operatorname{int} L$ is the union of a finite number of polyhedra.

It follows that the properties presented in this section of the closed convex hull of the union of a finite number of polyhedra are valid also for this special case. Thus by Theorem 3 the set $P \backslash L$ is polyhedral, and by Corollary 5 it is possible to optimize over $P \backslash L$ in polynomial time.

Note that also in this special case the topological closure is required in (6) in order to get a closed set. As an example consider polyhedra $P:=\left\{z \in \mathbb{R}^{2}: z \geq 0, z_{2} \leq 1\right\}$, and $L:=\left\{z \in \mathbb{R}^{2}: z \geq 0\right\}$.

Given general polyhedra $P$ and $L$, the polyhedron $P \backslash L$ may have an exponential number of facets. This may happen even when $L$ is the convex hull of two parallel and different hyperplanes, as shown in the following example.

Example 1 Let $P$ and $L$ be polyhedra in $\mathbb{R}^{n}$ defined as follows:

$$
\begin{aligned}
& P:=\left\{z \in \mathbb{R}^{n}:-1 \leq \sum_{j=1}^{n} z_{j} \leq 1,-1 \leq z_{i}-\sum_{\substack{j=1 \\
j \neq i}}^{n} z_{j} \leq 1, \quad i=1, \ldots, n\right\}, \\
& L:=\left\{z \in \mathbb{R}^{n}:-1 \leq \sum_{j=1}^{n} z_{j} \leq 1\right\} .
\end{aligned}
$$

Moreover consider the following two polyhedra:

$$
\begin{aligned}
& P^{1}:=P \cap\left\{z \in \mathbb{R}^{n}: \sum_{j=1}^{n} z_{j} \geq 1\right\}, \\
& P^{2}:=P \cap\left\{z \in \mathbb{R}^{n}: \sum_{j=1}^{n} z_{j} \leq-1\right\} .
\end{aligned}
$$

Clearly $P \backslash L=\overline{\operatorname{conv}}\left(P^{1} \cup P^{2}\right)$. It can be verified that the vertices of $P^{1}$ are the vectors $e^{1}, \ldots, e^{n}$ of the canonical basis of $\mathbb{R}^{n}$, while the vertices of $P^{2}$ are the vectors $-e^{1}, \ldots,-e^{n}$. It follows that $P \backslash L=\operatorname{conv}\left\{ \pm e^{1}, \ldots, \pm e^{n}\right\}$ is the "cross polytope" in dimension $n$. The cross polytope is described by $\sum_{i=1}^{n}\left|z_{i}\right| \leq 1$, and has $2^{n}$ facets, one for every orthant of $\mathbb{R}^{n}$ (see Fig. 4). 
Fig. 4 The cross polytope in dimension 3

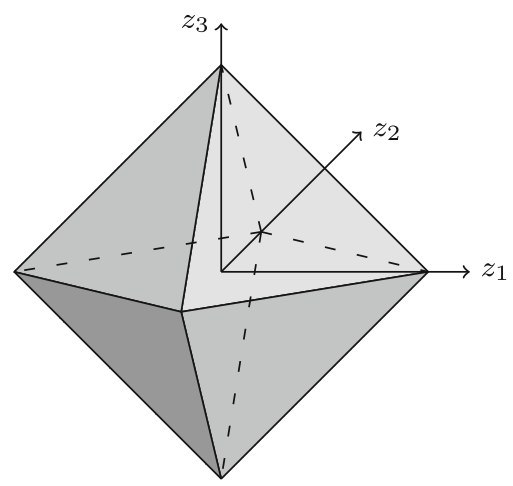

We recall that a polyhedron $S \subseteq \mathbb{R}^{p}$ is called a strip if $S=\left\{x \in \mathbb{R}^{p}: \beta \leq a x \leq\right.$ $\beta+1\}$ where $a \in \mathbb{Z}^{p}, \beta \in \mathbb{Z}$, and the greatest common divisor of the entries in $a$ is 1 . A slight modification of Example 1 shows that $P \backslash S$ may have an exponential number of facets even when $S$ is a strip.

Example 2 Let $P$ and $S$ be polyhedra in $\mathbb{R}^{n}$ defined as follows:

$$
\begin{aligned}
& P:=\left\{z \in \mathbb{R}^{n}: 0 \leq \sum_{j=1}^{n} z_{j} \leq 1, z \leq 1,(n-1) z_{i}+n \sum_{\substack{j=1 \\
j \neq i}}^{n} z_{j} \leq n, i=1, \ldots, n\right\}, \\
& S:=\left\{z \in \mathbb{R}^{n}: 0 \leq \sum_{j=1}^{n} z_{j} \leq 1\right\} .
\end{aligned}
$$

Moreover consider the following two polyhedra:

$$
\begin{aligned}
& P^{1}:=P \cap\left\{z \in \mathbb{R}^{n}: \sum_{j=1}^{n} z_{j} \geq 1\right\}, \\
& P^{2}:=P \cap\left\{z \in \mathbb{R}^{n}: \sum_{j=1}^{n} z_{j} \leq 0\right\} .
\end{aligned}
$$

Clearly $P \backslash S=\overline{\operatorname{conv}}\left(P^{1} \cup P^{2}\right)$. It can be verified that the vertices of $P^{1}$ are the vectors $e^{1}, \ldots, e^{n}$ of the canonical basis of $\mathbb{R}^{n}$, while the vertices of $P^{2}$ are the vectors $1-n e^{1}, \ldots, 1-n e^{n}$. It can be verified that $P \backslash S$ has exactly $2^{n}$ facets.

\subsection{Polyhedral relaxations}

Recent work has focused on trying to simplify the construction of $P \backslash L$. One way to do this is to obtain a characterization of $P \backslash L$ by considering, instead of a description 
of the polyhedron $P$, the descriptions of relaxations of $P$ that are defined by a subset of the inequalities defining $P$.

Let $A z \leq b$ be a system of inequalities defining a polyhedron $P$. A basic relaxation of $P$ is a polyhedral relaxation of $P$ that consists of the intersection of the half-spaces corresponding to at most $n$ linearly independent inequalities of the system $A z \leq b$. In what follows we denote by $\mathcal{R}(A, b)$ the family of the basic relaxations of $P$. Moreover, we denote by $\mathcal{R}^{k}(A, b)$ the family of the polyhedral relaxations of $P$ that consist of the intersection of the half-spaces corresponding to at most $k$ inequalities of the system $A z \leq b$.

Andersen et al. (2005) proved the following:

Theorem 7 Let $P=\left\{z \in \mathbb{R}^{n}: A z \leq b\right\}$ be a polyhedron, and let $S$ be a strip. Then

$$
P \backslash S=\bigcap_{R \in \mathcal{R}(A, b)} R \backslash S .
$$

A short proof of Theorem 7 has been recently provided by Dash et al. (2011d), and uses the equivalence between split cuts and MIR cuts.

Theorem 7 is very useful for constructing $P \backslash S$ when $S$ is a strip. In fact, given a basic relaxation $R$ of $P$ and any polyhedron $L$ in $\mathbb{R}^{n}$, the polyhedron $R \backslash L$ can be described by the system defining $R$ and at most one additional inequality (see for example Conforti et al. 2011a).

In the more general case where $L$ is a polyhedron with $h \geq 2$ facets, Conforti and Del Pia (2011) proved the following theorem:

Theorem 8 Let $P=\left\{z \in \mathbb{R}^{n}: A z \leq b\right\}$ and L be polyhedra in $\mathbb{R}^{n}$, and let $h \geq 2$ be the number of facets of $L$. Then

$$
P \backslash L=\bigcap_{R \in \mathcal{R}^{n(h-1)}(A, b)} R \backslash L .
$$

In Conforti and Del Pia (2011) it is also shown that (7) does not hold if one considers only polyhedral relaxations in $\mathcal{R}^{n(h-1)-1}(A, b)$.

Note that if $h \geq 3$, the polyhedral relaxations in $\mathcal{R}^{n(h-1)}(A, b)$ generally do not consist only of polyhedra described by linearly independent inequalities. One might wonder if it is sufficient to consider in (7) only polyhedral relaxations that consist of linearly independent inequalities. This does not generally hold true, as shown in Conforti and Del Pia (2011) for $h \geq 3$, and in Andersen et al. (2005) for $h=2$.

\section{Lattice-free polyhedra}

To get polyhedra contained in $P$ and that contain $P_{I}$, it is possible to use the set $P \backslash L$ introduced in (6). The set $P \backslash L$ is polyhedral by Theorem 3, and this implies that such an operation can be also used iteratively. The following is trivially true: 
Observation 9 Let $P$ and $L$ be polyhedra in $\mathbb{R}^{n}$. Then $P \backslash L \subseteq P$.

The question arises which polyhedra $L$ one should use in order to approximate $P_{I}$ sufficiently well. This first requires to characterize polyhedra $L$ such that $P_{I} \subseteq P \backslash L$ for every polyhedron $P$ in $\mathbb{R}^{p+q}$.

Given a polyhedron $L$, we say that $L$ is $\mathbb{Z}^{p} \times \mathbb{R}^{q}$-free if relint $L$ does not contain any point in $\mathbb{Z}^{p} \times \mathbb{R}^{q}$.

Observation 10 Let $L$ be a polyhedron in $\mathbb{R}^{p+q}$. Then $P_{I} \subseteq P \backslash L$ for every polyhedron $P$ in $\mathbb{R}^{p+q}$ if and only if $L$ is $\mathbb{Z}^{p} \times \mathbb{R}^{q}$-free.

Hence, given a polyhedron $P$ in $\mathbb{R}^{p+q}$, any $\mathbb{Z}^{p} \times \mathbb{R}^{q}$-free polyhedron $L$ can be used to get a polyhedron $P \backslash L$ such that $P_{I} \subseteq P \backslash L \subseteq P$.

The following easy observation points out the importance of the concept of inclusion when working with the convexification operation $P \backslash L$.

Observation 11 Let $P, L, L^{\prime}$ be polyhedra in $\mathbb{R}^{n}$ with $L \subseteq L^{\prime}$. Then $P \backslash L^{\prime} \subseteq P \backslash L$.

In what follows, a polyhedron $L \subseteq \mathbb{R}^{p}$ is said to be lattice-free if it is $\mathbb{Z}^{p}$-free. The following observation gives a link between $\mathbb{Z}^{p} \times \mathbb{R}^{q}$-free polyhedra in $\mathbb{R}^{p+q}$ and lattice-free polyhedra in $\mathbb{R}^{p}$. For its proof see Conforti et al. (2011a).

Observation 12 Let $L$ be a $\mathbb{Z}^{p} \times \mathbb{R}^{q}$-free polyhedron. Then $\operatorname{proj}_{x} L$ is a lattice-free polyhedron and $L \subseteq\left(\operatorname{proj}_{x} L\right) \times \mathbb{R}^{q}$.

Given polyhedra $P \subseteq \mathbb{R}^{p+q}$, and $L \subseteq \mathbb{R}^{p}$, we denote by

$$
P \backslash L:=P \backslash\left(L \times \mathbb{R}^{q}\right) .
$$

If we could use all lattice-free polyhedra algorithmically then we would be in good position to compute the mixed integer hull. In fact Jörg (2008, Lemma 3.5) and Conforti et al. (2010a) showed the following:

Theorem 13 Let $P$ be a rational polyhedron in $\mathbb{R}^{p+q}$, let $c z \leq \gamma$ be a rational inequality valid for $P_{I}$, and let $\mathcal{L}$ be the family of all lattice-free rational polyhedra. Then there exists $L \in \mathcal{L}$ such that $c z \leq \gamma$ is valid for $P \backslash L$.

Note that if $P$ is rational, by Theorem $1, P_{I}$ is a rational polyhedron. Thus there exist inequalities $c^{i} z \leq \gamma^{i}, i=1, \ldots, k$ such that $P_{I}=\left\{z \in \mathbb{R}^{p+q}: c^{i} z \leq \gamma^{i}, i=\right.$ $1, \ldots, k\}$. By Theorem 13, for every $i=1, \ldots, k$, there exists a lattice-free rational polyhedron $L^{i}$ such that $c^{i} z \leq \gamma^{i}$ is valid for $P \backslash L^{i}$. Hence

$$
P_{I}=\bigcap_{i=1}^{k}\left(P \backslash L^{i}\right) .
$$

This implies that, if $\mathcal{L}$ is the family of all lattice-free rational polyhedra,

$$
P_{I}=\bigcap_{L \in \mathcal{L}}(P \backslash L) .
$$


This representation result is mathematically interesting and has been the point of departure for research in the past years. However, from an algorithmic point of view, this representation of $P_{I}$ is of little use since there are infinitely many lattice-free rational polyhedra, thus their explicit description seems to be entirely intractable.

This motivates us to restrict the family of lattice-free rational polyhedra which we admit to manipulate the polyhedron $P$ in order to describe or approximate its mixed integer hull. In Sects. 3.1 and 3.2 we present some special families of lattice-free polyhedra that turn out to be useful to approximate $P_{I}$.

\subsection{Maximal lattice-free polyhedra}

Given a family $\mathcal{L}$ of polyhedra, $L \in \mathcal{L}$ is said to be maximal in $\mathcal{L}$ if for every $L^{\prime} \in \mathcal{L}$ with $L \subseteq L^{\prime}, L=L^{\prime}$. We refer to polyhedra that are maximal in the family of lattice-free polyhedra as maximal lattice-free.

The following observation is well-known, and follows for example from Bell (1977).

Observation 14 A maximal polyhedron in the family of lattice-free rational polyhedra is maximal lattice-free.

Observation 14 implies that the family of maximal polyhedra in the family of latticefree rational polyhedra coincides with the family of the rational polyhedra among maximal lattice-free polyhedra. By Observation 11, Theorem 13 is still valid if $\mathcal{L}$ denotes the family of rational maximal lattice-free polyhedra in $\mathbb{R}^{p}$.

It follows again from Bell (1977) that each rational maximal lattice-free polyhedron is full-dimensional. The following example shows that in Theorem 13 we need to consider all rational maximal lattice-free polyhedra in $\mathbb{R}^{p}$ :

Example 3 Let $L$ be a rational maximal lattice-free polyhedron in $\mathbb{R}^{p}$. $L$ is fulldimensional, thus it has nonempty interior. Let $\bar{x} \in$ int $L$, and consider the polyhedron $P:=\operatorname{conv}((L \times\{0\}) \cup\{(\bar{x}, 1)\})$ in $\mathbb{R}^{p+1}$. It is easy to see that the inequality $y \leq 0$ is valid for $P_{I}$ and that the only lattice-free polyhedron $L^{\prime}$ such that $y \leq 0$ is valid for $P \backslash L^{\prime}$ is $L^{\prime}=L$.

The following theorem characterizes full-dimensional maximal lattice-free polyhedra. For its proof see for example Lovász (1989).

Theorem 15 A full-dimensional polyhedron $L$ in $\mathbb{R}^{p}$ is maximal lattice-free if and only if it is lattice-free, and every facet of $L$ contains a lattice point in its relative interior.

The following interesting result is due to Bell (1977) and Scarf (1977).

Theorem 16 Any full-dimensional maximal lattice-free polyhedron in $\mathbb{R}^{p}$ has at most $2^{p}$ facets.

The following example shows that the bound $2^{p}$ given in Theorem 16 is tight. 
Example 4 Let $L$ be the cross polytope in $\mathbb{R}^{p}$, i.e. $L:=\left\{x \in \mathbb{R}^{p}: \sum_{i=1}^{p}\left|x_{i}\right| \leq 1\right\}$. The cross polytope is a rational maximal lattice-free polytope with exactly $2^{p}$ facets.

Theorem 16 implies that every rational maximal lattice-free polyhedron in $\mathbb{R}^{p}$ has at most $2^{p}$ facets. In any case, the family of rational maximal lattice-free polyhedra in $\mathbb{R}^{p}$ is extremely complicated and large, thus their explicit description is practically intractable.

As an example, using Theorems 15 and 16 it is easy to characterize the rational maximal lattice-free polyhedra in $\mathbb{R}^{2}$. These maximal lattice-free polyhedra are strips, triangles, and quadrilaterals.

Observation 17 The rational maximal lattice-free polyhedra in the plane are of three possible forms:

(i) a strip $\beta \leq a_{1} x_{1}+a_{2} x_{2} \leq \beta+1$ where $a_{1}$ and $a_{2}$ are coprime integers and $\beta$ is an integer,

(ii) a triangle with an integral point in the relative interior of each of its edges,

(iii) a quadrilateral with an integral point in the relative interior of each of its edges.

\subsection{Integral lattice-free polyhedra}

A polyhedron $L$ in $\mathbb{R}^{p}$ is said to be integral if $L=L_{I}$. In this section, we introduce a smaller family of lattice-free polyhedra, namely the family of integral polyhedra among lattice-free polyhedra. We call such polyhedra integral lattice-free polyhedra. This family is not as strong as the family of rational maximal lattice-free polyhedra, in the sense that it does not yield a result as strong as Theorem 13. However we will see later how such a family allows us to derive a result similar to Theorem 13 if we allow ourselves to recursively apply the convexification operation $P \backslash L$.

By Observation 11, for our purposes it is enough to consider the maximal polyhedra among the integral lattice-free polyhedra. We call such polyhedra maximal integral lattice-free polyhedra. We call an affine transformation that maps $\mathbb{Z}^{p}$ into $\mathbb{Z}^{p}$ an affine unimodular transformation. A recent result by Averkov et al. (2011) shows the following.

Theorem 18 For every $p \in \mathbb{N}$, the family of maximal integral lattice-free polyhedra in $\mathbb{R}^{p}$ is finite up to affine unimodular transformations.

The explicit description of the maximal integral lattice-free polyhedra in $\mathbb{R}^{1}$ and $\mathbb{R}^{2}$ is well-known, but for $p \geq 3$ such a description is complicated, and still unknown.

Other interesting polyhedra are the integral polyhedra among the maximal latticefree polyhedra. We call such polyhedra integral maximal lattice-free polyhedra. It is easy to see that in general, the family of the integral maximal lattice-free polyhedra is contained in the family of the maximal integral lattice-free polyhedra. It can be verified that in $\mathbb{R}^{p}$, for $p \in\{1,2\}$, the two families coincide. Nill and Ziegler (2011) showed that in $\mathbb{R}^{p}$, for $p \geq 4$, the two families are different. It is still unknown if the two families differ or not in $\mathbb{R}^{3}$. In $\mathbb{R}^{3}$ an explicit description of the integral maximal lattice-free polyhedra is given by Averkov et al. (2011). 


\section{Closures}

In this section we focus on the $\mathcal{L}$-closure $\mathcal{L}(P)$ of $P_{I}$, that is the intersection of many sets $P \backslash L$, where $L$ varies in a family $\mathcal{L}$ of lattice-free polyhedra. By intersecting many sets $P \backslash L$, we can further improve our ability to approximate the mixed integer hull with families of lattice-free polyhedra.

Let $P \subseteq \mathbb{R}^{p+q}$ be a polyhedron, and let $\mathcal{L}$ be a (potentially infinite) family of polyhedra in $\mathbb{R}^{p}$.

The $\mathcal{L}$-closure $\mathcal{L}(P)$ of $P$ is:

$$
\mathcal{L}(P):=\bigcap_{L \in \mathcal{L}} P \backslash L
$$

If all the polyhedra in $\mathcal{L}$ are lattice-free, it follows from Observation 10 that the set $\mathcal{L}(P)$ contains $P_{I}$ for every polyhedron $P$. Moreover, $\mathcal{L}(P)$ is the set of points that satisfy all disjunctive cuts corresponding to every polyhedron in $\mathcal{L}$.

We denote by $\mathcal{S}$ the family of all the strips, and we call the set $\mathcal{S}(P)$ the split closure of $P$. Clearly $\mathcal{S}(P)$ is the intersection of all the split cuts for $P$. We also denote by $\mathcal{I}$ the family of the maximal integral lattice-free polyhedra. We call the set $\mathcal{I}(P)$ the integral lattice-free closure of $P$. Since every strip is an integral lattice-free polyhedron, it follows that $\mathcal{I}(P) \subseteq \mathcal{S}(P)$ for every polyhedron $P$.

The split closure has a very interesting property (see Cook et al. 1990):

Observation 19 Let $P$ be a rational polyhedron. If $P \neq P_{I}$ then $\mathcal{S}(P) \subsetneq P$.

This means that if $P \neq P_{I}$ then $\mathcal{S}(P)$ is always a better approximation of $P_{I}$ than $P$. Clearly, since $\mathcal{I}(P) \subseteq \mathcal{S}(P)$ for every polyhedron $P$, also the integral lattice-free closure satisfies this property.

Moreover, given a minimal face $F$ of $P$ that contains no point in $\mathbb{Z}^{p} \times \mathbb{R}^{q}$, it is easy to construct a split cut that cuts off $F$ (see for example Cook et al. 1990).

While it is possible to optimize over $P \backslash L$ in polynomial time (see Corollary 5), optimizing over a closure is generally hard. In fact, Caprara and Letchford showed that, given a rational polyhedron $P$ in $\mathbb{R}^{p+q}$, it is NP-hard to optimize a linear function over $\mathcal{S}(P)$ (Caprara and Letchford 2003). This means that given a point in $P$ it is NP-hard to find a split cut that cuts it off or to show that no such split cut exists.

\subsection{Finite generation and polyhedrality}

Let $\mathcal{L}$ be a family of polyhedra, and let $P$ be a polyhedron. Although for every $L \in \mathcal{L}$, the set $P \backslash L$ is polyhedral by Theorem 3 , the set $\mathcal{L}(P)$ is not necessarily polyhedral, as it is the intersection of a potentially infinite number of polyhedra. An interesting research direction is to understand under which assumptions on the family $\mathcal{L}$ and on the polyhedron $P$, the set $\mathcal{L}(P)$ is polyhedral.

Given $\mathcal{L}$ and $P$, we say that the set $\mathcal{L}(P)$ is finitely generated if there exists a finite subset $\overline{\mathcal{L}}$ of $\mathcal{L}$ such that $\mathcal{L}(P)=\overline{\mathcal{L}}(P)$. Clearly if $\mathcal{L}(P)$ is finitely generated, then the set $\mathcal{L}(P)$ is polyhedral, as it is the intersection of a finite number of polyhedra. The opposite implication does not hold in general, as shown by the following example. 
Example 5 Let $P:=\{x \in \mathbb{R}: 0 \leq x \leq 1 / 2\}$, and let $\mathcal{L}:=\left\{L^{i}: i \in \mathbb{N}\right\}$, where $L^{i}:=\{x \in \mathbb{R}: 1 / i \leq x \leq 1\}$ for every $i \in \mathbb{N}$. It is easy to check that $\mathcal{L}(P)=\{0\}$, and that for every finite subset $\overline{\mathcal{L}}$ of $\mathcal{L}, \mathcal{L}(P) \subsetneq \overline{\mathcal{L}}(P)$.

The following easy observation characterizes whenever $\mathcal{L}(P)$ is finitely generated.

Observation 20 Let $\mathcal{L}$ be a family of polyhedra, and let $P$ be a polyhedron. Then $\mathcal{L}(P)$ is finitely generated if and only if there exists a system $c^{i} z \leq \gamma^{i}, i=1, \ldots, k$, such that $\mathcal{L}(P)=\left\{z \in \mathbb{R}^{n}: c^{i} z \leq \gamma^{i}, i=1, \ldots, k\right\}$ and for every $i=1, \ldots, k$ there exists $L^{i} \in \mathcal{L}$ such that $c^{i} z \leq \gamma^{i}$ is valid for $P \backslash L^{i}$.

Most of the results in literature showing that, under certain assumptions, the set $\mathcal{L}(P)$ is polyhedral, actually prove that $\mathcal{L}(P)$ is finitely generated. Cook et al. (1990) prove the following.

Theorem 21 For any rational polyhedron $P$, the set $\mathcal{S}(P)$ is finitely generated.

Other proofs of Theorem 21 appear in Andersen et al. (2005), Dash et al. (2007), Vielma (2007).

Theorem 21 states that the set $\mathcal{L}(P)$ is finitely generated for every rational polyhedron $P$, for a specific class $\mathcal{L}$. A more general research direction is to give sufficient conditions on a family $\mathcal{L}$ for the set $\mathcal{L}(P)$ to be finitely generated for every rational polyhedron $P$.

Note that every strip $L$ in $\mathcal{S}$ satisfies the following condition:

for every facet $F$ of $L$, the affine hull of $F$ contains integral points.

Many other interesting lattice-free polyhedra satisfy condition (8). For example, Theorem 15 shows that each maximal lattice-free polyhedron in $\mathbb{R}^{p}$ satisfies condition (8). Also each integral polyhedron in $\mathbb{R}^{p}$ satisfies condition (8).

Let $L$ be a full-dimensional lattice-free polyhedron that satisfies condition (8), and let $a^{i} x \leq \beta^{i}, i=1, \ldots, k$, be an irredundant system such that $L=\left\{x \in \mathbb{R}^{p}: a^{i} x \leq\right.$ $\left.\beta^{i}, i=1, \ldots, k\right\}$, and such that for every $i=1, \ldots, k, a^{i} \in \mathbb{Z}^{p}$ and the greatest common divisor of the entries in $a^{i}$ is 1 . We define the max-facet-width $w(L)$ of $L$ as

$$
w(L):=\max \left\{\beta^{i}-\min \left\{a^{i} x: x \in L\right\}: i=1, \ldots, k\right\} .
$$

(Note that for every $i=1, \ldots, k, a^{i} \in \mathbb{Z}^{p}$ and condition (8) imply that $\beta^{i} \in \mathbb{Z}$.)

Example 6 Consider the full-dimensional lattice-free polyhedron $L:=\left\{x \in \mathbb{R}_{+}^{2}\right.$ : $\left.x_{1}+x_{2} \leq 2\right\}$. $L$ satisfies condition (8), and it can be easily verified that its max-facetwidth is $w(L)=2$. See Fig. 5 .

Andersen et al. (2010) give sufficient conditions for a set $\mathcal{L}(P)$ to be finitely generated for every rational polyhedron $P$ :

Theorem 22 Let $\mathcal{L}$ be a family of rational maximal lattice-free polyhedra such that there exists $k \in \mathbb{N}$ with $w(L) \leq k$ for every $L \in \mathcal{L}$. Then for any rational polyhedron $P$, the set $\mathcal{L}(P)$ is finitely generated. 

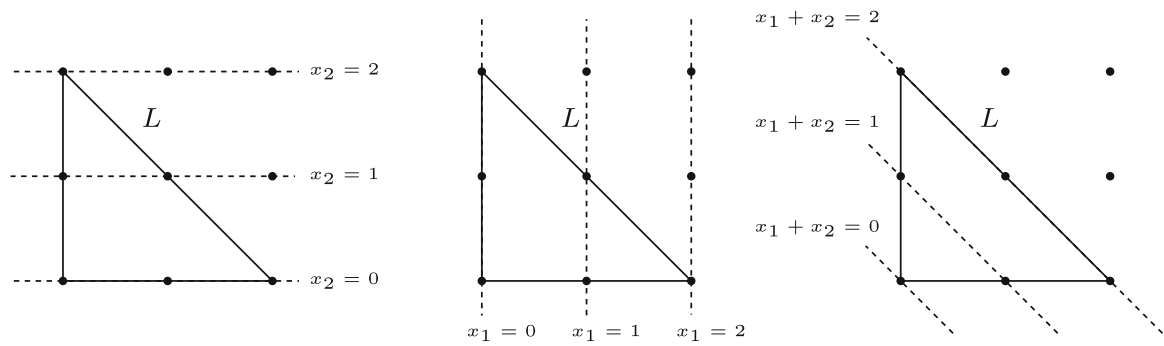

Fig. 5 The max-facet-width of $L:=\left\{x \in \mathbb{R}_{+}^{2}: x_{1}+x_{2} \leq 2\right\}$ is $w(L)=2$

Theorem 22 is stated only for maximal lattice-free polyhedra, but the proof in Andersen et al. (2010) works as well in a more general setting:

Theorem 23 Let $\mathcal{L}$ be a family of full-dimensional rational polyhedra that satisfy condition (8), and such that there exists $k \in \mathbb{N}$ with $w(L) \leq k$ for every $L \in \mathcal{L}$. Then for any rational polyhedron $P$, the set $\mathcal{L}(P)$ is finitely generated.

Del Pia and Weismantel (2011) use Theorem 23 to prove the following.

Theorem 24 For any rational polyhedron $P$, the set $\mathcal{I}(P)$ is finitely generated.

Even if for many interesting families of lattice-free polyhedra $\mathcal{L}$ the set $\mathcal{L}(P)$ is polyhedral for every rational polyhedron $P$, in most of the cases we cannot hope to get a full description of $\mathcal{L}(P)$. In fact, Example 2 shows that $\mathcal{L}(P)$ may have an exponential number of facets even if the family $\mathcal{L}$ consists of only one strip.

\subsection{Approximation of the mixed integer hull}

The sets $\mathcal{S}(P)$ and $\mathcal{I}(P)$ are generally only relaxations of $P_{I}$. It is easy to construct a polyhedron $P$ such that $\mathcal{S}(P) \neq P_{I}$, for example see Cook et al. (1990). For an example of a polyhedron $P$ such that $\mathcal{I}(P) \neq P_{I}$ see Del Pia and Weismantel (2011).

When considering mixed integer linear problems, even the split closure seems to approximate very well the mixed integer hull, from a practical point of view. In fact, Balas and Saxena (2008) performed experiments revealing that the split closure closes about $72 \%$ of the integrality gap on average on the well-known benchmark instances from MIPLIB 3.0.

Despite the fact that many computational tests have been performed to evaluate, for a given objective function, the gap between optimizing over the split closure versus the mixed integer hull, it still remains open to develop a mathematical approach that can analytically solve the following question: given a family $\mathcal{L}$, how well does $\mathcal{L}(P)$ approximate $P_{I}$ ?

In general $\mathcal{L}(P)$ does not equal $P_{I}$ for every polyhedron $P$. In this situation we aim at inventing a measure that is suited to quantify the distance of the sets $\mathcal{L}(P)$ and $P_{I}$.

An interesting measure of approximability is the strength measure introduced by Goemans (1995). Such measure can be applied to polyhedra of blocking type. 
A polyhedron $P$ in $\mathbb{R}^{n}$ is of blocking type if $P \subseteq \mathbb{R}_{+}^{n}$ and if $z^{\prime} \geq z \in P$ implies $z^{\prime} \in P$. For more details on polyhedra of blocking type see for example Schrijver (1986, Chapter 9). Given nonempty polyhedra of blocking type $P$ and $Q$ in $\mathbb{R}^{n}$ with $P \subseteq Q$, the strength of $P$ with respect to $Q$ is the minimum value of $\alpha \geq 1$ such that $Q \subseteq\left\{x \in \mathbb{R}^{n}: \alpha x \in P\right\}$. The strength measure is clearly a worst-case approximation measure.

Many recent papers have used the concept of strength to evaluate the quality of different types of closures. However, since the strength measure can be used only with polyhedra of blocking type, such studies mainly apply to the relaxed corner polyhedron that we introduced in Sect. 1.

In the relaxed corner polyhedron case, and when considering only two integer variables $(p=2)$, Basu et al. (2011a) showed that the split closure of $P$ is not always a good approximation of $P_{I}$. On the other hand they show that both $\mathcal{T}(P)$ and $\mathcal{Q}(P)$ are good approximations of $P_{I}$, where $\mathcal{T}$ is the family of maximal lattice-free triangles in $\mathbb{R}^{2}$, and $\mathcal{Q}$ is the family of maximal lattice-free quadrilaterals in $\mathbb{R}^{2}$. In the same settings, but using a probabilistic approach, Basu et al. (2010d) show that on average the split closure is a good approximation of $P_{I}$. Del Pia et al. (2011) show that the addition of a single triangle or quadrilateral inequality to the split closure becomes less likely to be beneficial the closer the lattice-free polyhedron looks like a strip, i.e. the closer its geometric width is to one.

We regard it as an interesting open question to find different measures of approximability that work for general polyhedra.

\section{Rounds of closures and convergence issues}

Given a rational polyhedron $P$, in order to get closer to $P_{I}$, in Sect. 3 we studied the object $P \backslash L$, for one single lattice-free polyhedron $L$. In Sect. 4 , in order to get a better approximation of $P_{I}$, we employed multiple lattice-free polyhedra from a family $\mathcal{L}$, and studied the more complicated set $\mathcal{L}(P)$. Also $\mathcal{L}(P)$ in many cases does not equal $P_{I}$. In particular this situation arises if $\mathcal{L}$ is either the family of the strips, or the family of the maximal integral lattice-free polyhedra. More generally, Example 3 shows that this may happen if $\mathcal{L}$ does not contain all the rational maximal lattice-free polyhedra.

This section studies the iterative application of the closure operation. Since we want to use the $\mathcal{L}$ operator recursively, we need $\mathcal{L}(P)$ to be a rational polyhedron for every rational polyhedron $P$. In this case, for every rational polyhedron $P$ in $\mathbb{R}^{p+q}$, and $i \in \mathbb{N}$ we can define the $i$-th $\mathcal{L}$-closure of $P$ as

$$
\mathcal{L}^{i}(P):=\mathcal{L}\left(\mathcal{L}^{i-1}(P)\right), \text { where } \mathcal{L}^{0}(P):=P
$$

Theorem 21 implies that, for every rational polyhedron $P$, the set $\mathcal{S}(P)$ is a rational polyhedron. We call the $i$-th $\mathcal{S}$-closure of $P$ simply the $i$-th split closure of $P$.

Cook et al. (1990) showed that there exist polyhedra $P$ such that $\mathcal{S}^{k}(P) \neq P_{I}$ for every $k \in \mathbb{N}$. The following is the example that they gave. 
Fig. 6 Cook, Kannan and Schrijver's example

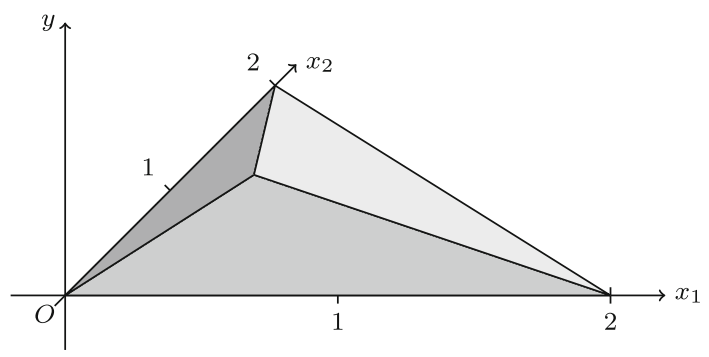

Example 7 Let $\mathbb{Q} \ni \epsilon>0$ and consider the polytope

$$
\begin{aligned}
P:= & \left\{(x, y) \in \mathbb{R}^{2} \times \mathbb{R}: y \geq 0, \epsilon x_{1}+\epsilon x_{2}+y \leq 2 \epsilon,\right. \\
& \left.-2 \epsilon x_{1}+y \leq 0,-2 \epsilon x_{2}+y \leq 0\right\} .
\end{aligned}
$$

(See Fig. 6.) It follows that $P$ is the convex hull of the four vectors

$$
(0,0,0),(2,0,0),(0,2,0),(1 / 2,1 / 2, \epsilon) .
$$

Since $x_{1}$ and $x_{2}$ are required to be integers while $y$ is not, it follows that $P_{I}$ is the convex hull of $(0,0,0),(2,0,0)$ and $(0,2,0)$, thus the inequality $y \leq 0$ is valid for $P_{I}$.

Now it can be shown that there exists $\mathbb{Q} \ni \epsilon^{1}>0$ such that $\left(1 / 2,1 / 2, \epsilon^{1}\right) \in \mathcal{S}(P)$. Hence $\mathcal{S}(P) \neq P_{I}$, and $\mathcal{S}(P)$ contains a polytope of the same form as $P$. So repeating the argument, for any $k \in \mathbb{N}$ the polytope $\mathcal{S}^{k}(P)$ contains the vector $\left(1 / 2,1 / 2, \epsilon^{k}\right)$ for some $\mathbb{Q} \ni \epsilon^{k}>0$. Therefore, we cannot obtain $P_{I}$ after a finite number of split closures.

Example 7 shows that split closures may never reach the mixed integer hull in a finite number of iterations. However in some special cases the split closures converge to the mixed integer hull in finite time. It follows from Chvátal (1973) and Schrijver (1980) that this applies to the pure integer case (i.e. $q=0$ ). This result also holds true for mixed 0,1 programs. In fact, Balas' sequential convexification theorem (Balas 1998) shows that in this case $\mathcal{S}^{p}(P)=P_{I}$, for every polyhedron $P$. Moreover, the bound is tight, meaning that it can happen that $\mathcal{S}^{p-1}(P) \neq P_{I}$ (Cornuéjols and $\mathrm{Li}$ 2002).

Owen and Mehrotra (2001) have shown that split closures can give an arbitrarily good approximation of $P_{I}$, when $P$ is a polytope. This result has been extended by Del Pia and Weismantel (2011) to general rational polyhedra. In this section we precisely state these results.

Let $\left\{\tilde{P}, P^{i}: i \in \mathbb{N}\right\}$ be a family of polyhedra such that $\tilde{P} \subseteq P^{i+1} \subseteq P^{i}$ for every $i \in \mathbb{N}$. We say that the sequence $\left\{P^{i}: i \in \mathbb{N}\right\}$ converges to $\tilde{P}$ if for every $\epsilon>0$, there exists $k \in \mathbb{N}$ such that $P^{k} \subseteq \tilde{P}+\epsilon B$, where $B$ denotes the unit ball in the corresponding space. The given definition of convergence is based on the Hausdorff distance, see Salinetti and Wets (1979, Section 3) for more details. Note that, if $\tilde{P}=\varnothing$, then it follows from the definition that there exists $k \in \mathbb{N}$ such that $P^{i}=\emptyset$ for all 
$i \geq k$. It is a well-known fact that if the sequence $\left\{P^{i}: i \in \mathbb{N}\right\}$ converges to $\tilde{P}$, then $\tilde{P}=\bigcap_{i \in \mathbb{N}} P^{i}$ (see for example Salinetti and Wets 1979, Theorem 2, Proposition 2).

There are however many other ways to define a notion of convergence for decreasing sequences of polyhedra (see for example Salinetti and Wets 1979). We now explain why the convergence that we defined makes sense in the context of mixed integer programming. The following observation can be found in Del Pia and Weismantel (2011):

Observation 25 Let $\left\{\tilde{P}, P^{i}: i \in \mathbb{N}\right\}$ be a family of polyhedra with $\tilde{P} \subseteq P^{i+1} \subseteq P^{i}$ for every $i \in \mathbb{N}$. Then the sequence $\left\{P^{i}: i \in \mathbb{N}\right\}$ converges to $\tilde{P}$ if and only if for every inequality $c z \leq \gamma$ valid for $\tilde{P}$, and every $\epsilon>0$, there exists $k \in \mathbb{N}$ such that $c z \leq \gamma+\epsilon$ is valid for $P^{k}$.

Now let $\left\{\tilde{P}, P^{i}: i \in \mathbb{N}\right\}$ be a family of polyhedra with $\tilde{P} \subseteq P^{i+1} \subseteq P^{i}$ for every $i \in \mathbb{N}$, such that the sequence $\left\{P^{i}: i \in \mathbb{N}\right\}$ converges to $\tilde{P}$. Consider the linear programming problem $\max \{c z: z \in \tilde{P}\}$, and let $\gamma$ be its optimal value. Since $c z \leq \gamma$ is valid for $\tilde{P}$, it follows from Observation 25 , that for every $\epsilon>0$, there exists $k \in \mathbb{N}$ such that $c z \leq \gamma+\epsilon$ is valid for $P^{k}$. Thus the limit of the sequence $\max \left\{c z: z \in P^{i}\right\}$ is the value $\gamma$. In other words, even if we do not know $\tilde{P}$, but we are able to construct the sequence $\left\{P^{i}: i \in \mathbb{N}\right\}$, we can approximate arbitrarily well the optimal value of any linear programming problem over $\tilde{P}$.

The following theorem was shown by Owen and Mehrotra (2001):

Theorem 26 For each polytope $P$, the sequence $\left\{\mathcal{S}^{i}(P): i \in \mathbb{N}\right\}$ converges to $P_{I}$.

Del Pia and Weismantel (2011) extended Theorem 26 to rational polyhedra:

Theorem 27 For each rational polyhedron $P$, the sequence $\left\{\mathcal{S}^{i}(P): i \in \mathbb{N}\right\}$ converges to $P_{I}$.

From the discussion in this section, we know that split cuts do not suffice alone to generate the mixed integer hull in a finite number of iterations. We definitely need to use a larger family of lattice-free polyhedra to obtain this goal. We next state the most important result of this section.

To this end, let us recall that $\mathcal{I}$ denotes the family of all maximal integral lattice-free polyhedra in $\mathbb{R}^{p}$. Theorem 24 implies that, for every rational polyhedron $P$, the set $\mathcal{I}(P)$ is a rational polyhedron. Thus for every rational polyhedron $P$ and every $i \in \mathbb{N}$ we can consider the $i$-th $\mathcal{I}$-closure of $P$, and we call it the $i$-th integral lattice-free closure of $P$. Del Pia and Weismantel (2011) showed the following:

Theorem 28 For each rational polyhedron $P$ there exists $k \in \mathbb{N}$ such that

$$
\mathcal{I}^{k}(P)=P_{I}
$$

Moreover, an easy generalization of Example 7 shows that if any polyhedron in $\mathcal{I}$ is omitted from the family, then the result does not hold (see for example Del Pia 2011). We believe that Theorem 28 ends a long open standing question. It remains though a major challenge to turn this structural knowledge into an algorithmic useful tool. 


\section{Rank of inequalities and cutting plane proofs}

Let $\mathcal{L}$ be a family of lattice-free polyhedra. We say that a lattice-free polyhedron $M$ has finite $\mathcal{L}$-rank if for every rational polyhedron $P$ there exists a sequence $L^{1}, \ldots, L^{k}$ of polyhedra in $\mathcal{L}$ such that

$$
\left(\cdots\left(\left(P \backslash L^{1}\right) \backslash L^{2}\right) \cdots\right) \backslash L^{k} \subseteq P \backslash M .
$$

The sense of this definition is the following: $M$ has finite $\mathcal{L}$-rank when, for every rational polyhedron $P$, a finite number of disjunctive cuts corresponding to lattice-free polyhedra in $\mathcal{L}$ can yield a relaxation of $P_{I}$ contained in the relaxation of $P_{I}$ that the $M$-disjunctive cuts can yield. Thus, if we are interested in what a cutting plane algorithm based on disjunctive cuts can achieve in finite time, $M$ has finite $\mathcal{L}$-rank if and only if a cutting plane algorithm based on the family $\mathcal{L} \cup\{M\}$ is always equivalent to an algorithm based only on the family $\mathcal{L}$.

Note that the given definition of rank is not the classic one. In fact, by directly extending the definition of finite Chvátal rank (see for example Schrijver 1986), we would say that a lattice-free polyhedron $M$ has finite $\mathcal{L}$-rank if for every rational polyhedron $P$ there exists $k \in \mathbb{N}$ such that $\mathcal{L}^{k}(P) \subseteq P \backslash M$. Clearly this classic definition requires that $\mathcal{L}(P)$ is polyhedral for every polyhedron $P$, while our definition does not need this assumption.

The following observation however shows that our definition of finite rank coincides with the classic one when $\mathcal{L}(P)$ is finitely generated for every rational polyhedron $P$. Notice that this property is satisfied by essentially all interesting families $\mathcal{L}$ of latticefree polyhedra, see Sect. 4.1.

Observation 29 Let $\mathcal{L}$ be a family offull-dimensional lattice-free polyhedra such that $\mathcal{L}(P)$ is finitely generated for every rational polyhedron $P$. A lattice-free polyhedron $M$ has finite $\mathcal{L}$-rank if and only if for every rational polyhedron $P$ there exists $k \in \mathbb{N}$ such that $\mathcal{L}^{k}(P) \subseteq P \backslash M$.

We now exhibit the proof of Observation 29 in order to familiarize the reader with the advanced and not entirely mature material presented in this section. We strongly believe that the discussion in this section can be extended further in the future and might lead to a very nice classification of lattice-free polyhedra.

Proof (of Observation 29) To prove necessity of the condition, assume that $M$ has finite $\mathcal{L}$-rank. Hence for every polyhedron $P$ there exists a sequence $L^{1}, \ldots, L^{k}$ of polyhedra in $\mathcal{L}$ such that

$$
\left(\cdots\left(\left(P \backslash L^{1}\right) \backslash L^{2}\right) \cdots\right) \backslash L^{k} \subseteq P \backslash M .
$$

By definition of $\mathcal{L}(P)$, we have that $\mathcal{L}(P) \subseteq P \backslash L^{i}$ for every $i=1, \ldots, k$, thus $\mathcal{L}^{k}(P) \subseteq\left(\cdots\left(\left(P \backslash L^{1}\right) \backslash L^{2}\right) \cdots\right) \backslash L^{k}$. It follows that $\mathcal{L}^{k}(P) \subseteq P \backslash M$.

To prove sufficiency of the condition we just need to show that for every polyhedron $P$, and for every $r \in \mathbb{N}$, there exists a sequence $L^{1}, \ldots, L^{k}$ of polyhedra in $\mathcal{L}$ such that 


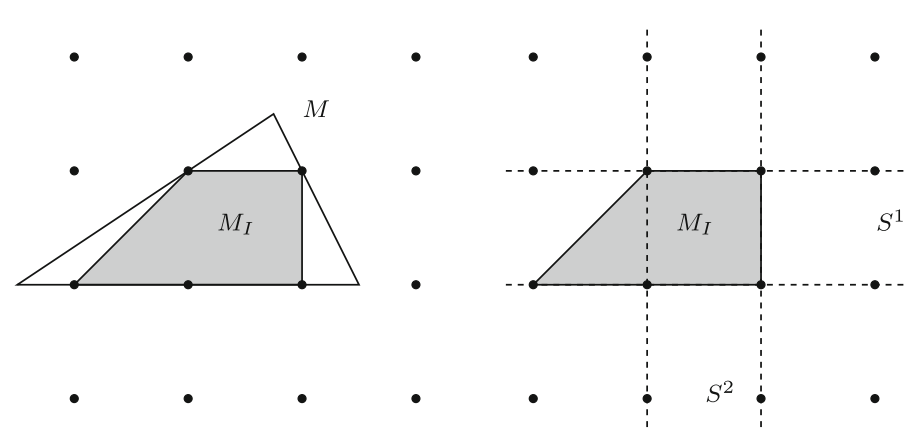

Fig. 7 On the left a polyhedron $M$ and its integer hull $M_{I}$. On the right, the strips $S^{1}$ and $S^{2}$ show that $M$ has the $\mathcal{S}$-inclusion property

$$
\left(\cdots\left(\left(P \backslash L^{1}\right) \backslash L^{2}\right) \cdots\right) \backslash L^{k} \subseteq \mathcal{L}^{r}(P) .
$$

So let $P$ be a polyhedron. We show this by induction on $r$. If $r=0$ the result is trivial. We now show the inductive step assuming $r \geq 1$. Since $\mathcal{L}(P)$ is a polyhedron, by induction hypothesis there exists a sequence $L^{1}, \ldots, L^{k}$ of polyhedra in $\mathcal{L}$ such that

$$
\left(\cdots\left(\left(\mathcal{L}(P) \backslash L^{1}\right) \backslash L^{2}\right) \cdots\right) \backslash L^{k} \subseteq \mathcal{L}^{r-1}(\mathcal{L}(P))=\mathcal{L}^{r}(P) .
$$

Since $\mathcal{L}(P)$ is finitely generated, there exists a finite subset $\overline{\mathcal{L}}=\left\{M^{j}: j=1, \ldots, h\right\}$ of $\mathcal{L}$ such that $\mathcal{L}(P)=\overline{\mathcal{L}}(P)$. Hence

$$
\left(\cdots\left(\left(P \backslash M^{1}\right) \backslash M^{2}\right) \cdots\right) \backslash M^{h} \subseteq \bigcap_{j=1}^{h} P \backslash M^{j}=\overline{\mathcal{L}}(P)=\mathcal{L}(P) .
$$

It follows that

$$
\left(\cdots\left(\left(\left[\left(\cdots\left(\left(P \backslash M^{1}\right) \backslash M^{2}\right) \cdots\right) \backslash M^{h}\right] \backslash L^{1}\right) \backslash L^{2}\right) \cdots\right) \backslash L^{k} \subseteq \mathcal{L}^{r}(P) .
$$

Del Pia (2011) gave a characterization of lattice-free polyhedra with finite $\mathcal{L}$-rank, for any family $\mathcal{L}$ of full-dimensional lattice-free polyhedra containing the strips. To be able to state the result precisely we need to give two definitions.

We say that a polyhedron $M \subseteq \mathbb{R}^{p}$ is $\mathcal{L}$-included if there exists $L \in \mathcal{L}$ such that relint $M \subseteq$ relint $L$. A polyhedron $M$ has the $\mathcal{L}$-inclusion property if every face $N$ of $M_{I}$ with relint $N \subseteq$ relint $M$ is $\mathcal{L}$-included. Note that one of the faces of $M_{I}$ is $M_{I}$ itself. See Figs. 7 and 8 for examples of polyhedra with or without the $\mathcal{S}$-inclusion property, where $\mathcal{S}$ is the family of all the strips.

Theorem 30 Let $\mathcal{L}$ be a family of full-dimensional lattice-free polyhedra containing the strips. A full-dimensional lattice-free polyhedron $M$ has finite $\mathcal{L}$-rank if and only if it has the $\mathcal{L}$-inclusion property.

Theorem 30 helps in the choice of candidate polyhedra $M$ to be added to a family $\mathcal{L}$ used for generating disjunctive cuts in a cutting plane algorithm: $M$ should 

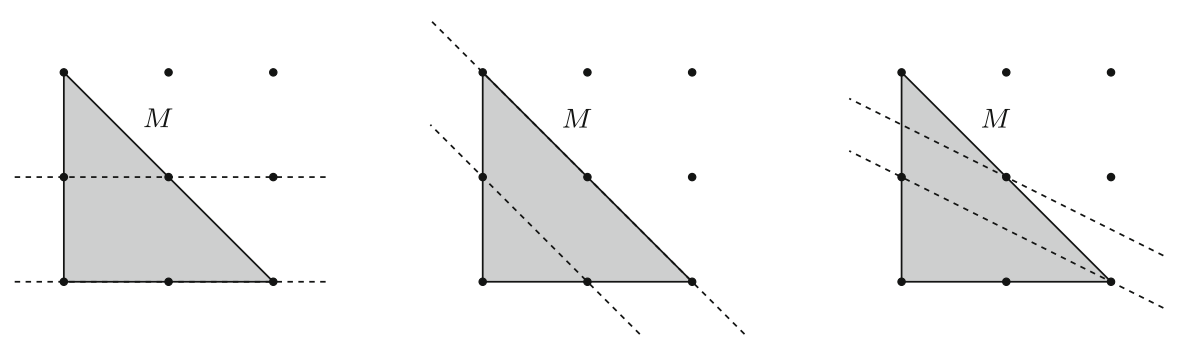

Fig. 8 A polyhedron $M=M_{I}$, and examples of strips $S$ with relint $M_{I} \nsubseteq \subseteq$ relint $S$. It can be checked that $M$ does not have the $\mathcal{S}$-inclusion property

be added to $\mathcal{L}$ if and only if it does not have the $\mathcal{L}$-inclusion property. Notice that the $\mathcal{L}$-inclusion property is a condition that can be checked aprioristically independently of the instance $P$.

We would like to point out that Theorem 30 has been discovered before by several authors in different special cases. Basu et al. (2011d) recently showed Theorem 30 in the relaxed corner polyhedron setting, when $\mathcal{L}$ is the family of the strips, and $M$ is bounded. Their results generalizes a theorem by Dey and Louveaux (2011) that holds when only two variables are required to be integer.

The concept of rank can also be adapted so as to apply to inequalities valid for $P_{I}$, once the polyhedron $P$ is fixed. Let $\mathcal{L}$ be a family of full-dimensional lattice-free polyhedra. Given a polyhedron $P$ and an inequality $c z \leq \gamma$ valid for $P_{I}$, we say that $c z \leq \gamma$ has finite $\mathcal{L}$-rank (with respect to $P$ ) if there exists a sequence $L^{1}, \ldots, L^{k}$ of polyhedra in $\mathcal{L}$ such that $c z \leq \gamma$ is valid for

$$
\left(\cdots\left(\left(P \backslash L^{1}\right) \backslash L^{2}\right) \cdots\right) \backslash L^{k} .
$$

Similar to Observation 29, the following result is easy to prove:

Observation 31 Let $\mathcal{L}$ be a family offull-dimensional lattice-free polyhedra such that $\mathcal{L}(P)$ is finitely generated for every rational polyhedron $P$, and let $P$ be a rational polyhedron. An inequality $c z \leq \gamma$ valid for $P_{I}$ has finite $\mathcal{L}$-rank if and only if there exists $k \in \mathbb{N}$ such that $c z \leq \gamma$ is valid for $\mathcal{L}^{k}(P)$.

A first corollary follows from Theorem 30, and characterizes the inequalities valid for $P_{I}$ that have finite $\mathcal{L}$-rank, for any family $\mathcal{L}$ of full-dimensional lattice-free polyhedra containing the strips.

Corollary 32 Let $\mathcal{L}$ be a family of full-dimensional lattice-free polyhedra containing the strips, let $P$ be a polyhedron, and let $c z \leq \gamma$ be an inequality valid for $P_{I}$. Then $c z \leq \gamma$ has finite $\mathcal{L}$-rank if and only if every face $M$ of $\operatorname{proj}_{x}\left\{z \in P_{I}: c z=\gamma\right\}$, with $M \cap \operatorname{proj}\{z \in P: c z>\gamma\} \neq \emptyset$, is $\mathcal{L}$-included.

Another consequence of Theorem 30 is the next corollary.

Corollary 33 Let $P \subseteq \mathbb{R}^{p+q}$ be a polyhedron, let $c z \leq \gamma$ be a valid inequality for $P_{I}$, and let $\mathcal{L}$ contain the strips and every full-dimensional integral lattice-free polyhedron $L$ with 
$\operatorname{dim} \operatorname{lin}$. space $L \geq p-\operatorname{dim} \underset{x}{\operatorname{proj}}\left\{z \in P_{I}: c z=\gamma\right\}$.

\section{Then $c z \leq \gamma$ has finite $\mathcal{L}$-rank.}

In particular, Corollary 33 states that if an inequality $c z \leq \gamma$ induces a vertex or an edge of $P_{I}$, then the inequality $c z \leq \gamma$ has finite split rank. This is an interesting observation because, given a random instance, most of the times (with probability 1) we are in this case. Accordingly, if $c z \leq \gamma$ induces a two-dimensional face of $P_{I}$, then it can be generated with a finite number of disjunctive cuts corresponding to full dimensional integral lattice-free polyhedra whose lineality space has dimension at least $p-2$. It is easy to see that such lattice-free polyhedra are all the strips and all the affine unimodular transformations of $\left\{x \in \mathbb{R}_{+}^{2}: x_{1}+x_{2} \leq 2\right\} \times \mathbb{R}^{p-2}$. In the other extreme case, if we define $\mathcal{I}$ to be the family of all the full-dimensional integral lattice-free polyhedra in $\mathbb{R}^{p}$, then Corollary 33 gives the statement of Theorem 28 .

It is still open whether analogues of Theorem 30 and its corollaries hold in the more general case where $\mathcal{L}$ is not required to contain all the strips.

In summary we believe that these results are a point of departure for defining a complexity measure for cutting planes in mixed integer programming. A first suggestion in this direction is given in Andersen et al. (2010). As in the pure integer case (see Schrijver 1986), we say that a sequence of linear inequalities $c^{1} z \leq \gamma^{1}, c^{2} z \leq$ $\gamma^{2}, \ldots, c^{k} z \leq \gamma^{k}$ is a $\mathcal{L}$-cutting plane proof of $c^{k} z \leq \gamma^{k}$ (from $P$ ), if for each $i=1, \ldots, k, c^{i} z \leq \gamma^{i}$ is a disjunctive cut for $P \cap\left\{x \in \mathbb{R}^{p+q}: c^{j} z \leq \gamma^{j}, j=\right.$ $1, \ldots, i-1\}$ corresponding to a lattice-free polyhedron in $\mathcal{L}$. The number $k$ will be called the length of the $\mathcal{L}$-cutting plane proof. It would be desirable to compute lower bounds on the length of $\mathcal{L}$-cutting plane proofs for special families of inequalities $c^{k} z \leq \gamma^{k}$ and for special classes of polyhedra $P$.

Assuming that there are enough students interested in questions of this kind, one could imagine that the approach discussed here can be refined in a way that it might result in a significantly better understanding of which lattice-free polyhedra can approximate the mixed integer hull for certain families of mixed integer programs.

We have only covered here parts of the recently emerging literature on the connections between mixed integer programming and lattice-free polyhedra. The reader that is interested in further developments of this topic is referred to the following papers: Averkov (2011), Balas and Margot (2011), Basu et al. (2010a,b,c, 2011b,c,e,f), Conforti et al. (2011b), Dash (2010), Dash et al. (2011a,b,c,e, 2010), Dey (2011), Dey and Wolsey (2008, 2010), Fukasawa and Günlük (2011), Morán and Dey (2011).

\section{References}

Andersen K, Cornuéjols G, Li Y (2005) Split closure and intersection cuts. Math Program A 102:457-493

Andersen K, Louveaux Q, Weismantel R (2010) An analysis of mixed integer linear sets based on lattice point free convex sets. Math Oper Res 35:233-256

Andersen K, Louveaux Q, Weismantel R, Wolsey LA (2007) Cutting planes from two rows of a simplex tableau. In: Proceedings of IPCO, lecture notes in computer science , vol 4513, pp 1-15

Averkov G (2011) On finite generation and infinite convergence of generalized closures from the theory of cutting planes (manuscript)

Averkov G, Wagner C, Weismantel R (2011) Maximal lattice-free polyhedra: finiteness and an explicit description in dimension three. Math Oper Res 36:721-742 
Balas E (1971) Intersection cuts — a new type of cutting planes for integer programming. Oper Res 19:19-39

Balas E (1979) Disjunctive programming. Ann Discret Math 5:3-51

Balas E (1985) Disjunctive programming and a hierarchy of relaxations for discrete optimization problems. SIAM J Algebraic Discret Methods 6:466-486

Balas E (1998) Disjunctive programming: properties of the convex hull of feasible points. GSIA management science research report MSRR 348, Carnegie Mellon University, 1974. Published as invited paper in Discrete Applied Mathematics 89:3-44

Balas E, Ceria S, Cornuéjols G (1993) A lift-and-project cutting plane algorithm for mixed 0-1 programs. Math Program 58:295-324

Balas E, Margot F (2011) Generalized intersection cuts and a new cut generating paradigm. Math Program A (to appear)

Balas E, Saxena A (2008) Optimizing over the split closure. Math Program A 113:219-240

Basu A, Campelo M, Conforti M, Cornuéjols G, Zambelli G (2010a) On lifting integer variables in minimal inequalities. In: Proceedings of IPCO, lecture notes in computer science, vol 6080, pp 85-95

Basu A, Conforti M, Cornuéjols G, Zambelli G (2010b) Maximal lattice-free convex sets in linear subspaces. Math Oper Res 35:704-720

Basu A, Conforti M, Cornuéjols G, Zambelli G (2010c) Minimal inequalities for an infinite relaxation of integer programs. SIAM J Discret Optim 24:158-168

Basu A, Cornuéjols G, Molinaro M (2010d) A probabilistic analysis of the strength of the split and triangle closures. Technical report

Basu A, Bonami P, Cornuéjols G, Margot F (2011a) On the relative strength of split, triangle and quadrilateral cuts. Math Program A 126:281-314

Basu A, Campelo M, Conforti M, Cornuéjols G, Zambelli G (2011b) Unique lifting of integer variables in minimal inequalities (manuscript)

Basu A, Cornuéjols G, Köppe M (2011c) Unique minimal liftings for simplicial polytopes (manuscript)

Basu A, Cornuéjols G, Margot F (2011d) Intersection cuts with infinite split rank. Math Oper Res (to appear)

Basu A, Cornuéjols G, Zambelli G (2011e) Convex sets and minimal sublinear functions. J Convex Anal 18(2):427-432

Basu A, Hildebrand R, Köppe M (2011f) Algorithmic and complexity results for cutting planes derived from maximal lattice-free convex sets (manuscript)

Bell DE (1977) A theorem concerning the integer lattice. Stud Appl Math 56:187-188

Benoy F, King A, Mesnard F (2005) Computing convex hulls with a linear solver. Theory Pract Logic Program 5(1\&2):259-271

Caprara A, Letchford AN (2003) On the separation of split cuts and related inequalities. Math Program B 94:279-294

Chvátal V (1973) Edmonds polytopes and a hierarchy of combinatorial problems. Discret Math 4:305-337

Conforti M, Cornuéjols G, Zambelli G (2010a) Eqivalence between intersection cuts and the corner polyhedron. Oper Res Lett 38:153-155

Conforti M, Cornuéjols G, Zambelli G (2010b) Polyhedral approaches to mixed integer linear programming. In: Juenger M, Liebling T, Naddef D, Pulleyblank W, Reinelt G, Rinaldi G, Wolsey L (eds) 50 years of integer programming 1958-2008: from the early years to the state-of-the-art. Springer, Berlin pp 334-384

Conforti M, Cornuéjols G, Zambelli G (2011a) Corner polyhedron and intersection cuts. Surv Oper Res Manag Sci 16:105-120

Conforti M, Cornuéjols G, Zambelli G (2011b) A geometric perspective on lifting. Oper Res 59:569-577

Conforti M, Del Pia A (2011) Disjunctive programming and relaxations of polyhedra. (manuscript)

Cook WJ, Kannan R, Schrijver A (1990) Chvátal closures for mixed integer programming problems. Math Program 47:155-174

Cornuéjols G, Li Y (2002) On the rank of mixed 0, 1 polyhedra. Math Program A 91:391-397

Dash S (2010) On the complexity of cutting-plane proofs using split cuts. Ope Res Lett 38:109-114

Dash S, Günlük O, Lodi A (2007) On the MIR closure of polyhedra. In: Proceedings of IPCO, lecture notes in computer science, vol 4513, pp 337-351

Dash S, Günlük O, Lodi A (2010) MIR closures of polyhedral sets. Math Program A 121:33-60

Dash S, Dey SS, Günlük O (2011a) On mixed-integer sets with two integer variables. Oper Res Lett 39: 305-309

Dash S, Dey SS, Günlük O (2011b) Two dimensional lattice-free cuts and asymmetric disjunctions for mixed-integer polyhedra. Math Program A (to appear) 
Dash S, Dobbs N, Günlük O, Nowicki T, Swirszcz G (2011c) Lattice-free sets, branching disjunctions, and mixed-integer programming (manuscript)

Dash S, Günlük O, Raack C (2011d) A note on the MIR closure and basic relaxations of polyhedra. Oper Res Lett 39:198-199

Dash S, Günlük O, Vielma JP (2011e) Computational experiments with cross and crooked cross cuts (manuscript)

Del Pia A (2011) On the rank of disjunctive cuts. Math Oper Res (to appear)

Del Pia A, Wagner C, Weismantel R (2011) A probabilistic comparison of the strength of split, triangle, and quadrilateral cuts. Oper Res Lett 39:234-240

Del Pia A, Weismantel R (2011) On convergence in mixed integer programming. Math Program A (to appear)

Dey SS (2011) A note on the split rank of intersection cuts. Math Program A 130:107-124

Dey SS, Louveaux Q (2011) Split rank of triangle and quadrilateral inequalities. Math Oper Res 36:432-461

Dey SS, Wolsey LA (2008) Lifting integer variables in minimal inequalities corresponding to lattice-free triangles. In: Proceedings of IPCO, lecture notes in computer science, vol 5035, pp 463-475

Dey SS, Wolsey LA (2010) Two row mixed-integer cuts via lifting. Math Program B 124:143-174

Fukasawa R, Günlük O (2011) Strengthening lattice-free cuts using non-negativity. Discret Optim 8: 229-245

Goemans MX (1995) Worst-case comparison of valid inequalities for the TSP. Math Program 69:335-349

Gomory RE (1963) An algorithm for integer solutions to linear programs. In: Graves RL, Wolfe P (eds) Recent advances in mathematical programming. McGraw-Hill, New York pp 269-302

Gomory RE (1965) On the relation between integer and non-integer solutions to linear programs. In: Proceedings of the National Academy of Sciences, vol 53, pp 260-265

Gomory RE (1969) Some polyhedra related to combinatorial problems. Linear Algebra Appl 2:451-558

Jörg M (2008) $k$-disjunctive cuts and cutting plane algorithms for general mixed integer linear programs. Ph.D. thesis, Technische Universität München, München

Khachiyan LG (1979) A polynomial algorithm in linear programming (in russian). Doklady Akademii Nauk SSSR 244:1093-1096 (English translation: Soviet Mathematics Doklady 20:191-194)

Khachiyan LG (1980) Polynomial algorithms in linear programming (in russian). Zhurnal Vychislitel'noi Matematiki i Matematicheskoi Fiziki 20:51-68. (English translation: U.S.S.R. Computational Mathematics and Mathematical Physics 20:53-72)

Lovász L (1989) Geometry of numbers and integer programming. In: Iri M, Tanabe K (eds) Mathematical programming: recent developments and applications. Kluwer, Dordrecht pp 177-201

Marchand H, Wolsey LA (2001) Aggregation and mixed integer rounding to solve MIPs. Oper Res 49: 363-371

Meyer RR (1974) On the existence of optimal solutions to integer and mixed-integer programming problems. Math Program 7:223-235

Morán R, Dey SS (2011) On maximal $S$-free convex sets. SIAM J Discret Math 25:379-393

Nemhauser GL, Wolsey LA (1990) A recursive procedure to generate all cuts for 0-1 mixed integer programs. Math Program 46:379-390

Nill B, Ziegler GM (2011) Projecting lattice polytopes without interior lattice points. Math Oper Res 36:462-467

Owen JH, Mehrotra S. (2001) A disjunctive cutting plane procedure for general mixed integer linear programs. Math Program A 89:437-448

Rockafellar RT (1970) Convex analysis. Princeton University Press, Princeton

Salinetti G, Wets RJ-B (1979) On the convergence of sequences of convex sets in finite dimensions. Soc Ind Appl Math 21:18-33

Scarf HE (1977) An observation on the structure of production sets with indivisiblities. Proc Natl Acad Sci USA 74:3637-3641

Schrijver A (1980) On cutting planes. Ann Discret Math 9:291-296

Schrijver A (1986) Theory of Linear and Integer Programming. Wiley, Chichester

Vielma JP (2007) A constructive characterization of the split closure of a mixed integer linear program. Oper Res Lett 35:29-35 